\title{
Magnetic Dynamics Studied by Time-Resolved Electron Microscopy
}

\author{
F. Carbone $^{1}$, R. Jayaraman ${ }^{1}$, Y. Murooka ${ }^{1}$, G.F. Mancini ${ }^{1}$, E. Baldini ${ }^{1}$, P. Huang ${ }^{2}$, M. \\ Cantoni $^{3}$, A. Magrez ${ }^{4}$, D. McGrouther ${ }^{5}$, T. Giamarchi ${ }^{6}$, H. M. Rønnow ${ }^{2}$ \\ 1. Laboratory for Ultrafast Microscopy and Electron Scattering, Ecole Polytechnique Fédérale \\ de Lausanne, CH-1015 Lausanne, Switzerland \\ 2. Laboratory for Quantum Magnetism, Ecole Polytechnique Fédérale de Lausanne, \\ Switzerland \\ 3. Centre Interdisciplinaire de Microscopie Electronique, CIME, Ecole Polytechnique \\ Fédérale de Lausanne, CH-1015 Lausanne, Switzerland \\ 4. Competence in Research of Electronically Advanced Materials, Ecole Polytechnique \\ Fédérale de Lausanne, CH-1015 Lausanne, Switzerland \\ 5. School of Physics and Astronomy, Kelvin Building, University of Glasgow G12 8QQ \\ Scotland \\ 6. Departement de Physique de la Matière Condensée, University of Geneva, Switzerland \\ email: fabrizio.carbone@epfl.ch
}

Keywords: magnetic dynamics, time-resolved electron microscopy, cryo-microscopy

For future technology, it is necessary to increase the data density of storage media, and to advance functionality of the various devices. Magnetism at the nanoscale is particularly promising for developing magnetic data storage, magnetic switching, spintronics, and $\mathrm{THz}$ technology.

While to date magnetic properties have mainly been observed and characterized statically, it has now become essential to monitor and capture their fast dynamics. The dynamics are based on fundamental and characteristic magnetic processes such as domain wall motion, skyrmion lattice formation, magnetic damping, vortex motions, spin-orbit coupling. These occur at their characteristic time scales spreading from $m s$ to fs. On the other hand, nanostructures can play essential roles for advancing functionality such as nanodot, hetero-structures, surface, defects, grain boundaries. Therefore it is often essential to directly monitor the localized nanoprocesses while responding to external stimulations such as electric/magnetic field, electron radiation, and temperature. For this purpose, we have developed time-resolved transmission electron microscopy (TEM), which was recently extended to the fs domain [1]. In contrast to other techniques such as Magnetic Force Microscopy, Holography, spin-polarized SEM, our approach can access time-resolved dynamics of bulk behaviors that happens between ms and fs. A pulsed laser was introduced externally both at the cathode and the sample. Photoelectrons are emitted from the cathode as a pulse train, and accelerated toward the sample to $200 \mathrm{kV}$. The dynamics can be induced by the laser pulse, and can be monitored at the fs time scale in a pump (laser)-probe (electron) geometry. ms dynamics can be recorder with a TV-rate camera or CCD camera, combined with an electron energy filter. We also extended our other capabilities for controlling the sample's temperature down to liquid helium temperature. With this cryo-TEM, for example, vortexes in superconductors MgB2 were revealed [2]. Furthermore, high quality crystals with unique properties are synthesized and were precisely characterized. Here, we will describe the dynamical role of disorder in a large and flat thin film of $\mathrm{Cu}_{2} \mathrm{OSeO}_{3}$, exhibiting a skyrmion phase in an insulating material [3]. A $150 \mathrm{~nm}$ thin film was prepared by Focused Ion Beam; particular care was devoted into obtaining a large $(7000 \mathrm{~nm})$ and flat sample. In Fig. 1, a Lorentz microscopy image of our sample is shown. This micrograph was recorded in our JEOL JEM2100 modified for ultrafast operation. The sample was cooled down to $7 \mathrm{~K}$ and Lorentz microscopy was performed in Fresnel mode by controlling the field produced by the microscope objective lens. A field of $400 \mathrm{G}$ was applied to the material and the skyrmion phase was induced. The image shows the regular distribution of over 50000 skyrmions. The effect of thermal and beam-irradiation induced fluctuations will be described showing the impact that disorder has on the skyrmion 
lattice orientation, and providing a unique point of view on the topology of this magnetic ground state.

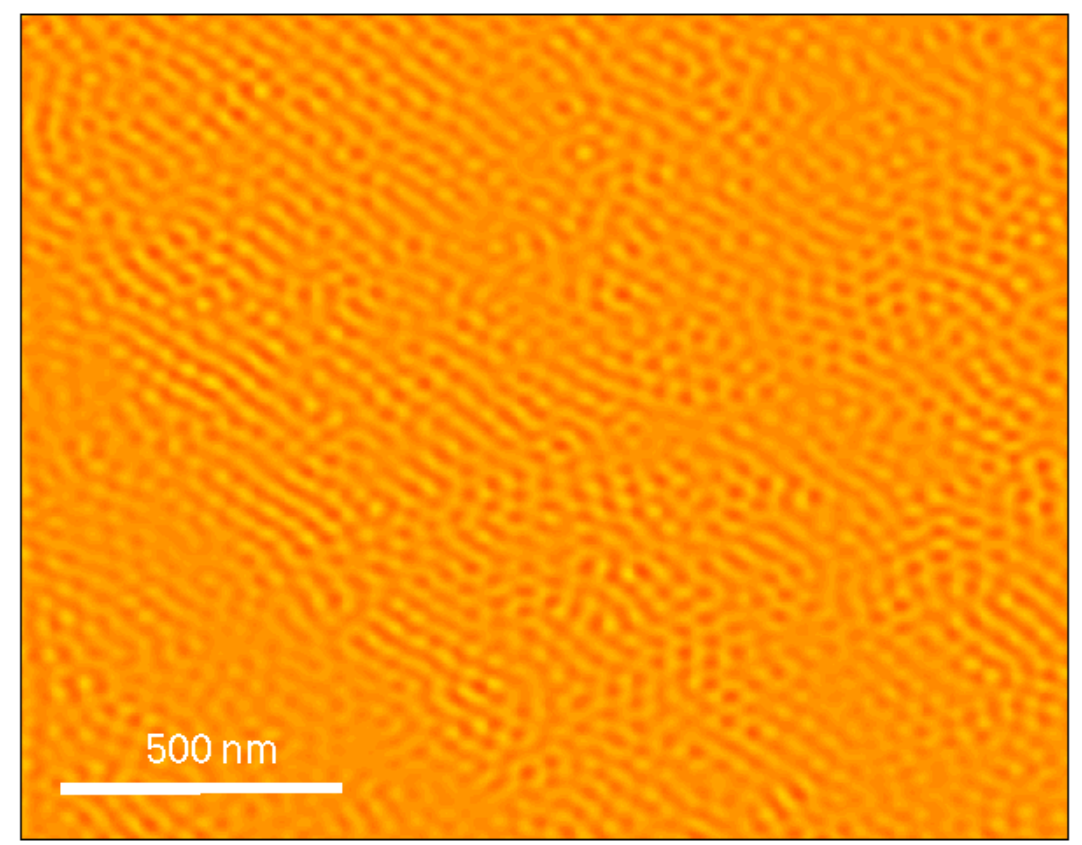

Fig. 1 Cryo-Lorentz TEM image of the skyrmion lattice forming within the helical domains in $\mathrm{Cu}_{2} \mathrm{OSeO}_{3}$. The applied field is $400 \mathrm{G}$ and the base temperature was $7 \mathrm{~K}$. The thin film was thinned down to $150 \mathrm{~nm}$.

\section{References:}

[1] L Piazza, et al., Chemical Physics 423 (2013), p. 79.

[2] M J G Cottet, et al., Phys. Rev. B 88 (2013), p. 014505.

[3] A A Omrani, et al., Phys. Rev. B 89 (2014), p. 064406.

[4] The authors gratefully acknowledge funding from an ERC starting grant. 